\title{
ORIGINAL
}

\section{Increasing evidence-based interventions in patients with acute infections in a resource-limited setting: a before-and-after feasibility trial in Gitwe, Rwanda}

Olivier Urayeneza ${ }^{1,2}$, Pierre Mujyarugamba', Zacharie Rukemba', Vincent Nyiringabo' ${ }^{1}$, Polyphile Ntihinyurwa', John I. Baelani ${ }^{3}$, Arthur Kwizera ${ }^{4}$, Danstan Bagenda ${ }^{5}$, Mervyn Mer ${ }^{6,7}$, Ndidiamaka Musa $^{8}$, Julia T. Hoffman ${ }^{5}$, Ashok Mudgapalli ${ }^{5}$, Austin M. Porter ${ }^{5}$, Niranjan Kissoon ${ }^{9}$, Hanno Ulmer ${ }^{10}$, Lori A. Harmon ${ }^{11}$, Joseph C. Farmer ${ }^{12}$, Martin W. Dünser ${ }^{13^{*}}$, Andrew J. Patterson ${ }^{5}$ and for the Sepsis in Resource-Limited Nations Workgroup of the Surviving Sepsis Campaign

(c) 2018 SCCM and ESICM

\begin{abstract}
Objective: To evaluate whether a focused education program and implementation of a treatment bundle increases the rate of early evidence-based interventions in patients with acute infections.
\end{abstract}

Design: Single-center, prospective, before-and-after feasibility trial.

Setting: Emergency department of a sub-Saharan African district hospital.

Patients: Patients $>28$ days of life admitted to the study hospital for an acute infection.

Interventions: The trial had three phases (each of 4 months). Interventions took place during the second (educational program followed by implementation of the treatment bundle) and third (provision of resources to implement treatment bundle) phases.

Measurements and main results: Demographic, clinical, and laboratory data were collected at study enrollment; 24, 48, and $72 \mathrm{~h}$ after hospital admission; and at discharge. A total of 1594 patients were enrolled (pre-intervention, $\mathrm{n}=661$; intervention $\mathrm{I}, \mathrm{n}=531$; intervention $\mathrm{I}, \mathrm{n}=402$ ). The rate of early evidence-based interventions per patient during Intervention Phase I was greater than during the pre-intervention phase (74 \pm 17 vs. $79 \pm 15 \%, p<0.001)$. No difference was detected when data were compared between Intervention Phases I and II (79 \pm 15 vs. $80 \pm 15 \%$, $p=0.58$ ). No differences in the incidence of blood transfusion (pre-intervention, $6 \%$; intervention I, $7 \%$; intervention II, $7 \%$ ) or severe adverse events in the first $24 \mathrm{~h}$ (allergic reactions: pre-intervention, $0.2 \%$; intervention I, 0\%; intervention II, $0 \%$; respiratory failure: pre-intervention, $2 \%$; intervention I, 2\%; intervention II, 2\%; acute renal failure: pre-intervention, 2\%; intervention I, 2\%; intervention II, 1\%) were observed.

*Correspondence: Martin.Duenser@i-med.ac.at

${ }^{13}$ Department of Anesthesiology and Intensive Care Medicine, Kepler University Hospital and Johannes Kepler University Linz, Linz, Austria

Full author information is available at the end of the article

This article is being published simultaneously in Critical Care Medicine (https://doi.org/10.1097/CCM.0000000000003227) and Intensive Care Medicine.

\section{实 Springer}


Conclusions: Our results indicate that a focused education program and implementation of an infection treatment bundle in clinical practice increased the rate of early evidence-based interventions in patients with acute infections (mostly malaria) admitted to a sub-Saharan African district hospital. Provision of material resources did not further increase this rate. While no safety issues were detected, this could be related to the very low disease severity of the enrolled patient population (http://www.clinicaltrials.gov: NCT02697513).

Keywords: Infection, Education, Bundle, Evidence-based intervention, Africa, Safety, Sepsis

\section{Introduction}

Infection and sepsis are among the leading causes of death worldwide. The annual burden of sepsis in highincome countries is estimated to be nearly $2.4-2.8$ million cases [1]. Mortality associated with sepsis is approximately $20-30 \%$ [2]. The impact of infection and sepsis on middle- and low-income countries is even greater. For example, 90\% of deaths worldwide from pneumonia, meningitis, and other infections occur in middle- and low-income countries [3]. Approximately $60 \%$ of the 7 million global deaths in neonates and infants annually are attributable to infection, with the majority of cases occurring in middle- and low-income countries [4]. Recommended intensive care interventions for the management of patients with sepsis [5] may be impractical, unaffordable, and possibly even harmful when implemented in middle- and low-income countries [6-9]. Consequently, early recognition and appropriate treatment of acute infection is essential.

The Sepsis in Resource-Limited Settings Workgroup of the Surviving Sepsis Campaign hypothesized that, in the low-income country of Rwanda, the rate of evidence-based interventions per patient during the first $6 \mathrm{~h}$ after hospital admission for patients experiencing acute infections would be higher after two interventions: (1) a focused infection education program, including the introduction of a simple infection bundle, and (2) provision of material resources to ensure that supplies are always immediately available for management of acute infection. We conducted this feasibility and safety trial at the rural Gitwe District Hospital and eight referring health centers to test our hypothesis that a focused education program and introduction of an infection treatment bundle would increase the rate of early evidence-based interventions in patients with acute infections.

\section{Materials and methods Trial design}

This study was designed as an investigator-initiated, single-center, prospective, before-and-after feasibility trial applying a step-wedge approach. The protocol of this trial was pre-published (http://www.clinicaltrials. gov; NCT02697513). It was reviewed and approved by the National Health and Research Committee as well as the Rwanda National Ethics Committee (no. 007/ RNEC/2016). The study was conducted in accordance with ethical principles of the Declaration of Helsinki. Written informed consent was obtained from all study patients or parents/next of kin of children before study enrollment. Consent forms were translated into Kinyarwanda. The trial was conducted at the adult and pediatric emergency department of Gitwe District Hospital as well as eight referring health centers in the Ruhango District, Southern Province, Rwanda. The manuscript was prepared according to a modified CONSORT statement (Electronic Supplementary Material).

\section{Gitwe District Hospital}

Gitwe District Hospital serves a population of approximately 160,000 and has 200 beds, one emergency department with separate adult and pediatric admission zones, two operating theaters, one laboratory (rapid diagnostic tests for HIV and tuberculosis available, malaria diagnosed by thick drop microscopy, no facilities for blood chemistry or microbiologic cultures), one radiography and one sonography machine. No intensive care or highdependency services are available. The hospital includes medical, surgical, pediatric, and gynecologic/obstetric departments. Surgical services are provided only on selected days. Patients with emergency conditions requiring urgent surgical interventions are usually transferred to the next-level hospital. Between the hours of 5:00 p.m. and 7:00 a.m., patients are directly managed by one oncall general practitioner and several in-house nurses and nurse assistants. The referring health centers are staffed by nurses and nurse assistants. Transfers over dirt roads between the health centers and Gitwe District Hospital occur mainly by four-wheel-drive vehicles equipped for ambulance service. Transfers are mostly limited to daylight hours.

\section{Patients}

Patients who were admitted to Gitwe District Hospital for a suspected or confirmed acute infectious disease (independent of the presence of sepsis) were eligible for study inclusion. Exclusion criteria were age $<28$ days of 
life, known allergy to any of the study drugs, limited therapy due to terminal disease, or refusal to provide written informed consent.

\section{Interventions}

The pre-intervention phase (March 14, 2016, until July $17,2016)$ consisted of acute infection incidence data collection. During this phase, there were no study-related interventions and treatment of study patients was determined by the attending physician. Clinical treatment guidelines for adults and children have been published by the Rwanda Ministry of Health [10] and are widely applied across the country. Intervention Phase I (July 18, 2016, until November 21, 2016) was immediately preceded by 1.5 -day education sessions focused on prevention and recognition of infection, as well as infection treatment bundles for adults and children (Fig. 1; course materials summarized in Figure S1 in the Electronic Supplementary Material). These education sessions took place over a 5-day period (July 11-15, 2016). One hundred sixty-three healthcare professionals (including nurses and physicians) from Gitwe District Hospital and the eight referring health centers participated in the education sessions. Education materials were locally oriented and printed by a community vendor. Pre-education session tests revealed knowledge gaps among the participating healthcare professionals with regard to recognition and management of acute infections. Post-education session tests demonstrated a significant reduction in these knowledge gaps. For instance, correct responses on a 15-question test improved from a mean of 7.8 (SD 3.4) before the training sessions to 13.7 (SD 2) after the sessions ( $p<0.01$, paired Student $t$ test). Intervention Phase I began on July 18,2016 , with implementation of pediatric $(<15$ years) and adult acute infection treatment bundles (Fig. 1). Intervention Phase II was initiated on November 21,2016 , and involved provision of material resources to ensure that supplies were consistently available to implement the infection treatment bundles at all study sites. For logistic reasons, oxygen could not be provided to the referring health centers or during transfer to Gitwe District Hospital.

\begin{tabular}{|c|c|c|c|c|c|c|}
\hline $\begin{array}{l}\text { Surviving } \\
\text { Sepsis": } \\
\text { Campaign : }\end{array}$ & \multicolumn{2}{|c|}{ ACUTE INFECTION ADULTS } & $\begin{array}{l}\text { Surviving } \\
\text { Sepsis": } \\
\text { Campaign : }\end{array}$ & \multicolumn{2}{|c|}{ ACUTE INFECTION CHILDREN } & $\begin{array}{l}\text { Sitwe } \\
\text { ospital }\end{array}$ \\
\hline \multirow{2}{*}{\multicolumn{3}{|c|}{$\begin{array}{l}\text { DANGER SIGNS: } \\
\square \text { altered mental state } \\
\square \text { respiratory distress } \\
\square \text { BPsys }<90 \mathrm{mmHg} \\
\text { OR cap refill } \geq 4 \mathrm{sec}\end{array}$}} & \multicolumn{4}{|c|}{ 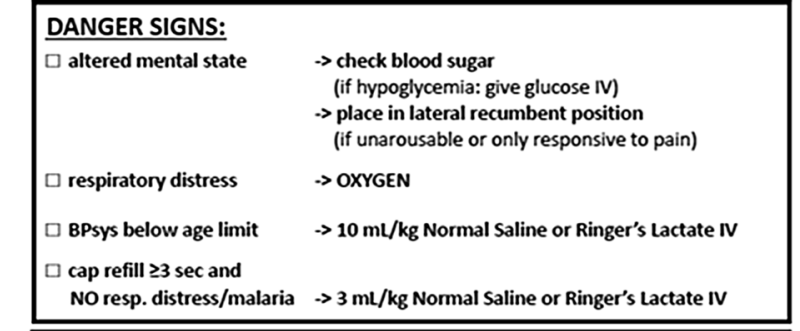 } \\
\hline & & & \multicolumn{4}{|c|}{ 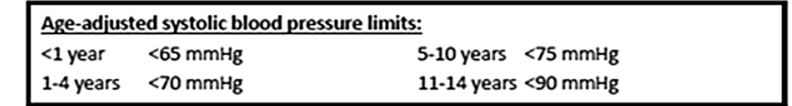 } \\
\hline \multicolumn{2}{|c|}{ BACTERIAL INFECTION } & & \multicolumn{4}{|c|}{ (when indicated) take LAB tests \pm rapid diagnostic test/blood smear } \\
\hline \multirow{2}{*}{\multicolumn{2}{|c|}{$\begin{array}{l}\square \text { start ANTIBIOTIC } \\
\text { uncomplicated infection: PO } \\
\text { severe infection/no PO tolerance: IV } \\
\text { (empiric IV antibiotics backside) } \\
\square \text { if abscess, abdominal, puerperal } \\
\text { or joint infection: consider SURGERY }\end{array}$}} & C Uncomnlicated Malaria. & \multicolumn{2}{|c|}{ BACTERIAL INFECTION } & \multicolumn{2}{|l|}{ MALARIA } \\
\hline & & $\begin{array}{l}\text { Artemether/Lumefantrine } 4 \text { Tablets PO } \\
\text { q Severe Malaria: Artesunate IV } \\
\text { (dose scheme backside) } \\
\square \text { Cerebral Malaria: Artesunate IV } \\
\text { (dose scheme backside) PLUS } \\
\text { Chloramphenicol } 2 \times 1 \mathrm{~g} \mathrm{IV}\end{array}$ & $\begin{array}{l}\square \text { start A } \\
\text { uncom } \\
\text { severe } \\
\text { (empir } \\
\square \text { if absc } \\
\text { or join }\end{array}$ & $\begin{array}{l}\text { IIBIOTIC } \\
\text { licated infection: PO } \\
\text { ffection/no PO tolerance: IV } \\
\text { IV antibiotics backside) } \\
\text { s, abdominal, puerperal } \\
\text { ffection: consider SURGERY }\end{array}$ & $\begin{array}{l}\text { Uncomplicated Malaria: } \\
\begin{array}{l}\text { Artemether/Lumefantrine PO } \\
\text { (dose scheme backside) }\end{array} \\
\text { Severe Malaria: } \\
\square \text { Artesunate IV } \\
\text { (dose scheme backside) } \\
\text { PLUS Cefotaxime } 50 \mathrm{mg} / \mathrm{kg} \text { IV }\end{array}$ & \\
\hline \multicolumn{3}{|c|}{$\begin{array}{l}\square \text { if suspicion of relevant anemia: check hemoglobin and transfuse if necessary } \\
\square \text { if HIV positive, malnutrition, previous Tb infection or close contact to persons } \\
\text { suffering from Tb: Tb screening as per local policy }\end{array}$} & \multicolumn{4}{|c|}{$\begin{array}{l}\text { if suspicion of relevant anemia: check hemoglobin and transfuse if necessary } \\
\text { if HIV positive, malnutrition, previous Tb infection or close contact to persons } \\
\text { suffering from Tb: Tb screening }\end{array}$} \\
\hline \multicolumn{3}{|c|}{$\begin{array}{l}\text { NO over-hydration NO steroids } \\
\text { NO diuretics to treat oliguria }\end{array}$} & \multicolumn{4}{|c|}{$\begin{array}{l}\text { NO over-hydration NO steroids } \\
\text { NO diuretics to treat oliguria }\end{array}$} \\
\hline
\end{tabular}

Fig. 1 Infection treatment bundles for adults and children implemented during the intervention phases of this study 


\section{Data Documentation and Processing}

Throughout all three study phases, three research assistants who were not otherwise involved in patient care and worked on a rotation schedule of $24 \mathrm{~h}$ per day, 7 days per week, were present in the emergency department of Gitwe District Hospital. These research assistants screened patients for study eligibility and collected data for all study patients. They utilized a standardized documentation sheet (Figure S2 Electronic Supplementary Material). All patients included in the trial were reevaluated at 24, 48, and $72 \mathrm{~h}$ after hospital admission, and at hospital discharge. Anonymized data were entered into an electronic database (REDCap) [11] and securely stored at the University of Nebraska Medical Center in Omaha, Nebraska, USA. Before data analysis, the database records underwent repeated checks to detect documentation and/or entry errors.

\section{Definitions}

For this study, sepsis was defined according to the 1991 American College of Chest Physicians/Society of Critical Care Medicine criteria [12]. Since blood tests to determine the full Sequential Organ Failure Assessment (SOFA) score were not available at any of the study sites in Rwanda, the quick SOFA (qSOFA) score (calculated from data collected at admission to the emergency department of Gitwe District Hospital) was used to identify study patients with an increased risk of death as suggested by the Sepsis-3 study group [13]. In children ( $<15$ years), sepsis was diagnosed if the child had a suspected or proven infection with any one of the following criteria: body temperature $<36{ }^{\circ} \mathrm{C}$, body temperature $>38^{\circ} \mathrm{C}$ plus altered mental status, body temperature $>38^{\circ} \mathrm{C}$ plus respiratory distress, body temperature $>38^{\circ} \mathrm{C}$ plus a history of not feeding, or body temperature $>38^{\circ} \mathrm{C}$ plus convulsions [14]. Relevant other definitions are summarized in Table S1 of the Electronic Supplementary Material.

\section{Outcome measures}

The primary outcome measure for this investigation was the difference in the rate of evidence-based interventions per study patient during the first six hours after admission to the emergency department of Gitwe District Hospital during the pre-intervention phase and Intervention Phase I. Evidence-based interventions were defined as: (1) initiation of antimicrobial therapy; (2) surgical source control; (3) blood glucose measurement and IV glucose administration (in cases of altered mental state and hypoglycemia, respectively); (4) placement of patient in recovery position (in cases of unresponsiveness to touch and/or pain); (5) oxygen administration (in cases of respiratory distress); and (6) fluid administration (in cases of systemic hypoperfusion). The primary outcome was assessed at $6 \mathrm{~h}$ after study patients were admitted to the emergency department of Gitwe District Hospital.

Secondary outcome measures for this investigation included the difference in the rate of evidence-based interventions (defined and assessed as above) per study patient during the first $6 \mathrm{~h}$ after admission to the emergency department of Gitwe District Hospital during Intervention Phases I and II. Also included were the difference in the rate of evidence-based interventions (defined and assessed as above) at referring health centers over the three study phases. In addition, secondary outcome measures included differences in the incidence of newly occurring sepsis during the first $72 \mathrm{~h}$ after hospital admission over the three study phases (assessed at 24-h intervals during the first $72 \mathrm{~h}$ after hospital admission) as well as difference in hospital mortality rate (assessed at hospital discharge) over the three study phases. Further secondary outcome measures included differences in the incidence of pulmonary edema, allergic reactions, and acute renal failure during the first $24 \mathrm{~h}$ after hospital admission (assessed $24 \mathrm{~h}$ after hospital admission), as well as differences in the need for blood transfusion over the three study phases (assessed at hospital discharge).

\section{Statistical analysis}

The primary null hypothesis was: a focused education program and introduction of a simple acute infection treatment bundle would not influence the rate of early, evidence-based interventions. The study was designed as a superiority trial with $80 \%$ power to detect a 15 -percentage-point difference in the rate of early evidence-based interventions per patient (e.g., 50-65\%) at an alpha error of 5\%. These assumptions were based on 2012-2014 statistics from Gitwe District Hospital suggesting that approximately 1200 patients annually are admitted to the hospital for acute infections.

One interim analysis was performed on September 27, 2016. During this interim analysis, the incidence of predefined adverse events (allergic reaction, pulmonary edema, allergic reactions, and acute renal failure during the first $24 \mathrm{~h}$ after hospital admission) and hospital mortality were compared between the pre-intervention phase and Intervention Phase I. O'Brien-Fleming boundaries were applied. No differences were observed in any of these parameters. Consequently, patient recruitment continued.

Following locking and plausibility control of the database, statistical analyses were performed, applying the intention-to-treat principle and using the PASW statistical software package (IBM SPSS Statistics 20; IBM). Normality assumption of all continuous data was tested using Shapiro-Wilk tests. All variables were approximately normally distributed except for the duration of 
symptoms before hospital admission, the number of infectious foci, and blood glucose level at hospital admission. These variables are presented as median values with interquartile ranges. In view of the fact that all datasets for primary and secondary outcome measures were complete, no statistical methods were used to compensate for missing values. Descriptive statistical methods were used to report demographic, clinical, and outcome data. Comparisons between groups were conducted using the unpaired Student $t$-test or Chi square test, as appropriate. In addition to the main analysis, the primary outcome measure was analyzed separately for the following a priori defined subgroups (including tests for interaction): children ( $<15$ years), adults, patients with malaria, and patients referred from health centers. In view of the low number of patients with known or newly diagnosed HIV infection $(\mathrm{n}=33)$, we discarded the planned subgroup analysis that included only patients positive for HIV. We hypothesized that significant differences in the primary outcome measure between the pre-intervention phase and Intervention Phase I would also be detectable in these subgroups (without differences between subgroups). In a post hoc analysis, an unadjusted logistic regression analysis was performed to assess whether the number of evidence-based interventions (independent variable) was associated with hospital survival (dependent variable). Data are presented as mean values $\pm S D$ or absolute values with percentages, if not otherwise indicated. We considered $p$ values $<0.05$ to indicate statistical significance.

\section{Results}

During the 12-month study period (March 14, 2016, until March 13, 2017), 1594 patients were enrolled in the trial (Fig. 2). No patient withdrew consent or was lost to follow-up until hospital discharge. Table 1 summarizes the characteristics of study patients recruited during each study phase.

\section{Primary and Secondary Outcomes}

The incidence of evidence-based interventions per patient during the first $6 \mathrm{~h}$ after hospital admission for treatment of infection during Intervention Phase I was significantly greater than the incidence of evidence-based interventions during the pre-intervention phase $(74 \pm 17$ vs. $79 \pm 15 \%, p<0.001)$. No difference in the incidence of evidence-based interventions per patient was detected between Intervention Phases I and II. The incidence of antimicrobial therapy initiation and the incidence of fluid resuscitation at the health centers were both significantly greater when data from the two intervention phases were compared to the pre-intervention phase. We observed no differences in the incidence of new sepsis during the first $72 \mathrm{~h}$ after hospital admission or the hospital survival rate over the three study phases (Table 2).

\section{Safety outcomes}

The mean amount of IV fluid administered to study patients presenting with signs of systemic hypoperfusion over the first $6 \mathrm{~h}$ of the study was significantly greater during the two intervention phases compared to the preintervention phase (pre-intervention: $32 \pm 21 \mathrm{~mL} / \mathrm{kg} / 6 \mathrm{~h}$,

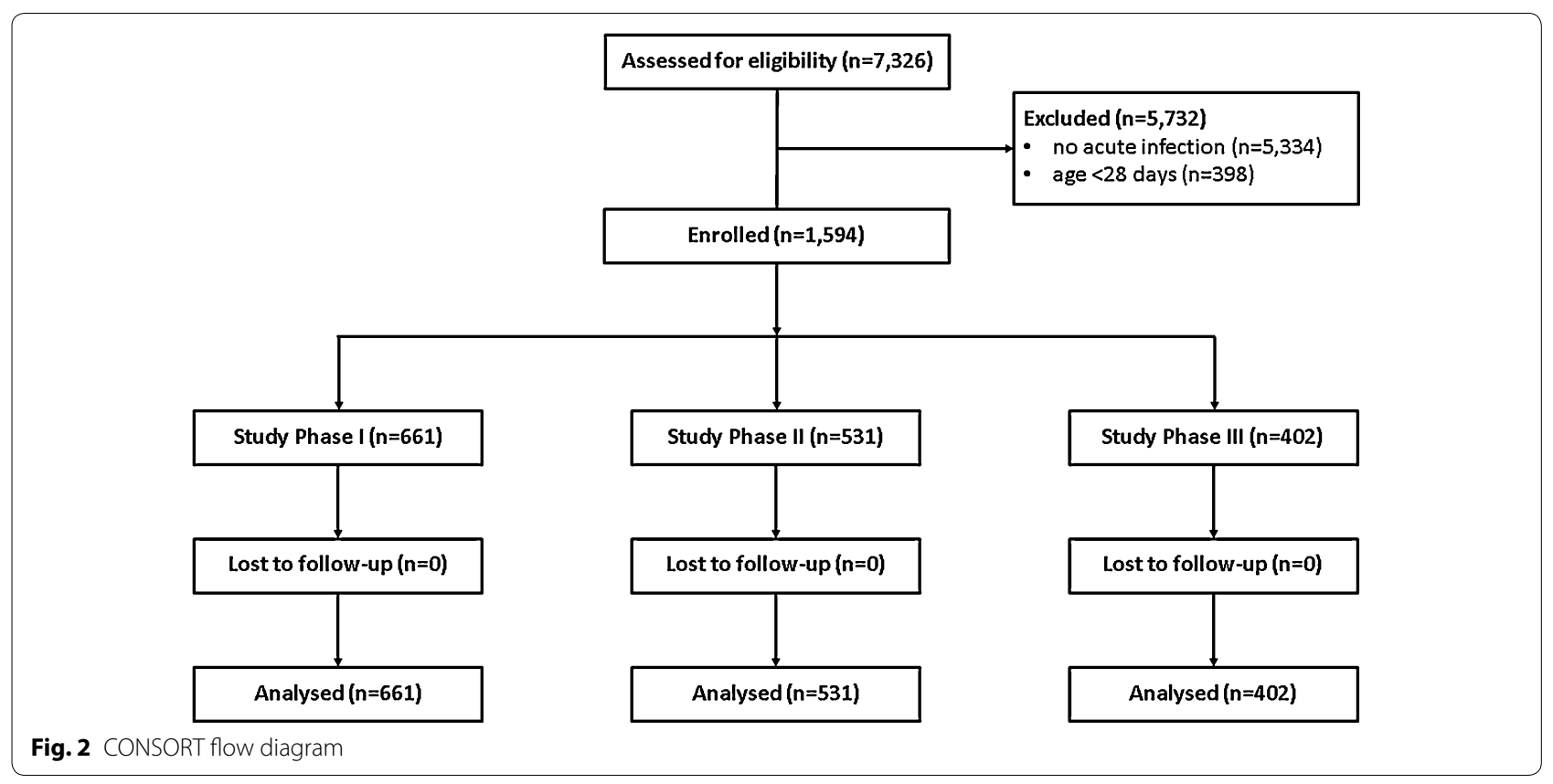


Table 1 Characteristics of patients enrolled during each study phase

\begin{tabular}{|c|c|c|c|}
\hline & Pre-intervention phase & Intervention phase I & Intervention phase II \\
\hline$n$ & 661 & 531 & 402 \\
\hline Age (years) & $20 \pm 23$ & $17 \pm 21$ & $18 \pm 22$ \\
\hline Age $<15$ years $[n(\%)]$ & $386(58.4)$ & $335(63.1)$ & $253(62.9)$ \\
\hline Male gender [n (\%)] & $274(41.5)$ & $271(51)$ & $191(47.5)$ \\
\hline \multicolumn{4}{|l|}{ HIV status [n (\%)] } \\
\hline Negative/unknown & $641(97)$ & $526(99.1)$ & $394(98)$ \\
\hline Positive on HAART & $16(2.4)$ & $4(0.8)$ & $8(2)$ \\
\hline Positive not on HAART & $4(0.6)$ & $1(0.2)$ & 0 \\
\hline \multicolumn{4}{|l|}{ (Known) comorbidities [n (\%)] } \\
\hline Arterial hypertension & $10(1.5)$ & $8(1.5)$ & $5(1.2)$ \\
\hline Diabetes mellitus & $8(1.2)$ & 0 & $4(0.9)$ \\
\hline Gastritis & $6(0.9)$ & $9(1.7)$ & $5(1.2)$ \\
\hline Malnutrition & $6(0.9)$ & $5(0.9)$ & 0 \\
\hline Duration of symptoms before hospital admission (days) & $2(1-4)$ median (IQR) & $2(1-4)$ median (IQR) & $2(1-4.5)$ median (IQR) \\
\hline \multicolumn{4}{|l|}{ Self-medication [n (\%)] } \\
\hline Over-the-counter antimicrobials & $5(0.8)$ & $13(2.4)$ & $13(3.2)$ \\
\hline Herbal medicine & $8(1.2)$ & $19(3.6)$ & $11(2.7)$ \\
\hline Other over-the-counter drugs & $3(0.5)$ & $2(0.4)$ & $1(0.2)$ \\
\hline \multicolumn{4}{|l|}{ Recent history of $[n(\%)]$} \\
\hline Not feeding & $277(41.9)$ & $220(41.4)$ & $164(40.8)$ \\
\hline Convulsions & $120(18.2)$ & $123(23.2)$ & $103(25.6)$ \\
\hline \multicolumn{4}{|l|}{ Admission source $[n(\%)]$} \\
\hline Health center & $579(87.6)$ & $487(91.7)$ & $380(94.5)$ \\
\hline Home & $75(11.3)$ & $32(6)$ & $19(4.7)$ \\
\hline Other & $7(1.1)$ & $12(2.2)$ & $3(0.7)$ \\
\hline Infectious foci $(n)$ & 1 (1-2) median (IQR) & $2(1-2)$ median (IQR) & 1 (1-2) median (IQR) \\
\hline \multicolumn{4}{|l|}{ Infectious source $[n(\%)]$} \\
\hline Severe malaria & $387(58.5)$ & $319(60.1)$ & $252(62.7)$ \\
\hline Lungs & $214(32.4)$ & $111(20.9)$ & $80(19.9)$ \\
\hline Uncomplicated malaria & $129(19.5)$ & $130(24.5)$ & $96(23.9)$ \\
\hline Gastrointestinal & $107(16.2)$ & $120(22.6)$ & $70(17.4)$ \\
\hline Urinary tract & $65(9.8)$ & $43(8.1)$ & $42(10.4)$ \\
\hline Meningitis & $28(4.2)$ & $33(6.2)$ & $7(1.7)$ \\
\hline Skin/soft tissue & $9(1.4)$ & $9(1.7)$ & $9(2.2)$ \\
\hline Abdominal & $8(1.2)$ & $4(0.8)$ & $5(1.2)$ \\
\hline Puerperal & $4(0.6)$ & $7(1.3)$ & $3(0.7)$ \\
\hline Other $^{\mathrm{a}}$ & $20(3)$ & $44(8.3)$ & $9(2.2)$ \\
\hline PBSI/unknown & $4(0.6)$ & $41(7.7)$ & $66(16.4)$ \\
\hline \multicolumn{4}{|l|}{ Functional state at hospital admission $[n(\%)]^{b}$} \\
\hline Walked unassisted & $90(13.6)$ & $63(11.9)$ & $48(11.9)$ \\
\hline Walked assisted & $114(17.2)$ & $112(21.1)$ & $89(22.1)$ \\
\hline Could not walk & $259(39.2)$ & $169(31.8)$ & $125(31.1)$ \\
\hline \multicolumn{4}{|l|}{ Physiologic variables at hospital admission } \\
\hline Heart rate (bpm) & $105 \pm 23$ & $104 \pm 20$ & $104 \pm 20$ \\
\hline Respiratory rate (bpm) & $25 \pm 8$ & $25 \pm 8$ & $25 \pm 8$ \\
\hline Systolic blood pressure (mmHg) & $112 \pm 24$ & $104 \pm 20$ & $107 \pm 18$ \\
\hline Temperature $\left({ }^{\circ} \mathrm{C}\right)$ & $37.6 \pm 1.1$ & $37.6 \pm 1.2$ & $37.6 \pm 1.2$ \\
\hline Capillary refill time (s) & $1.9 \pm 0.9$ & $2.4 \pm 1$ & $2.2 \pm 1$ \\
\hline
\end{tabular}


Table 1 continued

\begin{tabular}{|c|c|c|c|}
\hline & Pre-intervention phase & Intervention phase I & Intervention phase II \\
\hline \multicolumn{4}{|l|}{ Laboratory values at hospital admission } \\
\hline Blood sugar (mg/dL) & 124 (96-158) Median (IQR) & 135 (111-181) Median (IQR) & 129 (107-159) Median (IQR) \\
\hline Hemoglobin (g/dL) & $11 \pm 3$ & $11 \pm 3$ & $11 \pm 3$ \\
\hline White cell count $(\mathrm{G} / \mathrm{L})$ & $10 \pm 12$ & $9 \pm 8$ & $9 \pm 6$ \\
\hline \multicolumn{4}{|l|}{ Danger signs at hospital admission $[n(\%)]$} \\
\hline Altered mental state & $123(18.6)$ & $75(14.1)$ & $49(12.2)$ \\
\hline Unresponsive or only responsive to pain & $59(8.9)$ & $32(6)$ & $30(7.5)$ \\
\hline Respiratory distress & $108(16.3)$ & $52(9.8)$ & $47(11.7)$ \\
\hline Systemic hypoperfusion & $64(9.7)$ & $82(15.4)$ & $59(14.7)$ \\
\hline Referred to next level hospital [n (\%)] & $18(2.7)$ & $14(2.6)$ & $14(3.5)$ \\
\hline Infection confirmed at hospital discharge $[n(\%)]^{c}$ & $580(87.7)$ & $475(89.5)$ & $357(88.8)$ \\
\hline Hospital length of stay (days) & $5 \pm 5$ & $4 \pm 4$ & $4 \pm 4$ \\
\hline Total hospital costs (1000 RWF) & $37 \pm 32$ & $31 \pm 23$ & $36 \pm 39$ \\
\hline
\end{tabular}

Data are given as mean values $\pm S D$, if not otherwise indicated

Datasets were complete for all variables except for: heart rate at admission (missing $n=41$ ), respiratory rate at admission (missing $n=29$ ), systolic blood pressure at admission (missing $n=974$ ), temperature at admission (missing $n=25)$, capillary refill time at admission $(\operatorname{missing} n=34)$, blood sugar (missing $n=1368)$, hemoglobin at admission (missing $n=156$ ), white cell count at admission (missing $n=405$ ), and total hospital costs (missing $n=8$ ). Note that laboratory values at hospital admission were taken at the discretion of the attending physician and based on availability of laboratory facilities. According to the treatment bundle, blood sugar was only required to be taken if the patient presented with an altered mental state

IQR interquartile range

a Other infectious sources included tonsillitis/pharyngitis, otitis, osteomyelitis, arthritis, hepatitis, syphilis, and toxoplasmosis

b In children $>2$ years

c "Infection confirmed at hospital discharge" refers to the number (percentage) in how many patients the initial diagnosis of an acute infectious disease was confirmed at hospital discharge

\section{Table 2 Secondary outcome measures}

\begin{tabular}{|c|c|c|c|c|}
\hline Outcome measures & Pre-intervention phase & Intervention phase I & Intervention phase II & $p$ value \\
\hline $\begin{array}{l}\text { Rate of evidence-based interventions per patient within } 6 \mathrm{~h} \text { after } \\
\text { hospital admission (\%) }\end{array}$ & & $79 \pm 15$ & $80 \pm 15$ & 0.58 \\
\hline Antimicrobials initiated by HCC [n (\%)] & $261(40)$ & $276(52)$ & $213(53)$ & $<0.001^{*}$ \\
\hline Fluid resuscitation initiated by HCC [n (\%)] & $75(11)$ & $125(24)$ & $83(21)$ & $<0.001^{*}$ \\
\hline New sepsis (ACCP/SCCM) within $72 \mathrm{~h}$ after admission [ $n(\%)]$ & $57(9)$ & $54(10)$ & $49(12)$ & 0.18 \\
\hline New qSOFA criteria within $72 \mathrm{~h}$ after admission [n (\%)] & $50(8)$ & $48(9)$ & $44(11)$ & 0.18 \\
\hline Hospital survival [n (\%)] & $635(96)$ & $514(97)$ & $385(96)$ & 0.51 \\
\hline
\end{tabular}

Data are given as mean values $\pm S D$, if not otherwise indicated

HCC healthcare centre

*Significant group difference (Chi ${ }^{2}$-test)

$\mathrm{n}=451$; Intervention $\mathrm{I}: 40 \pm 23 \mathrm{~mL} / \mathrm{kg} / 6 \mathrm{~h}, \mathrm{n}=389$; Intervention II: $39 \pm 22 \mathrm{~mL} / \mathrm{kg} / 6 \mathrm{~h}, \mathrm{n}=277 ; p<0.001)$. When data from all study phases were compared, we observed no differences between the incidence of severe adverse events in the first $24 \mathrm{~h}$ after hospital admission. Further, we observed no differences in the incidence of blood transfusion over the three study phases (Table 3). We observed similar results for all safety outcome measures when data from children and adults were compared.

\section{Subgroup and post hoc analyses}

The primary outcome measure was significantly different when data from the pre-intervention and Intervention Phase I were compared for all a priori defined subgroups. Interaction tests identified significant differences for the effect size between adults and children as well as between patients with and without malaria (co-) infection (Table 4). The unadjusted logistic regression analysis revealed a higher incidence of hospital survival as the number of evidence-based interventions implemented increased during the first $6 \mathrm{~h}$ after hospital 
Table 3 Incidences of serious adverse events and need for blood transfusion

\begin{tabular}{|c|c|c|c|}
\hline & Pre-intervention phase & Intervention phase I & Intervention phase II \\
\hline \multicolumn{4}{|l|}{ All patients } \\
\hline Allergic reaction (first 24 h) & $1(0.2 ; 0.004-0.8)$ & $0(0 ; 0-0.7)$ & $0(0 ; 0-0.9)$ \\
\hline Respiratory failure (first 24 h) & $16(2.4 ; 1.4-3.9)$ & $13(2.4 ; 1.3-4.2)$ & $6(1.5 ; 0.6-3.2)$ \\
\hline Acute renal failure (first 24 h) & $12(1.8 ; 0.9-3.2)$ & $11(2 ; 1-3.7)$ & $4(1 ; 0.3-2.5)$ \\
\hline Need for blood transfusion & $41(6 ; 4.5-8.3)$ & $35(6.6 ; 4.6-9)$ & $27(6.7 ; 4.4-9.6)$ \\
\hline \multicolumn{4}{|l|}{ Adults } \\
\hline Allergic reaction (first 24 h) & $1(0.4 ; 0.009-2)$ & $0(0 ; 0-1.9)$ & $0(0 ; 0-2.4)$ \\
\hline Respiratory failure (first 24 h) & $9(3.3 ; 1.5-6.1)$ & $8(4.1 ; 1.8-7.9)$ & $3(2 ; 0.4-5.8)$ \\
\hline Acute renal failure (first 24 h) & $12(4.4 ; 2.3-7.5)$ & $10(5.1 ; 2.5-9.2)$ & $4(2.7 ; 0.7-6.7)$ \\
\hline Need for blood transfusion & $15(5.5 ; 3.1-8.8)$ & $15(7.7 ; 4.3-12.3)$ & $10(6.7 ; 3.3-12)$ \\
\hline \multicolumn{4}{|l|}{ Children $(<15$ years) } \\
\hline Allergic reaction (first 24 h) & $0(0 ; 0-1)$ & $0(0 ; 0-1.1)$ & $0(0 ; 0-1.4)$ \\
\hline Respiratory failure (first 24 h) & $7(1.8 ; 0.7-3.7)$ & $5(1.5 ; 0.5-3.4)$ & $3(1.2 ; 0.2-3.4)$ \\
\hline Acute renal failure (first 24 h) & $0(0 ; 0-1)$ & $1(0.3 ; 0.008-1.7)$ & $0(0 ; 0-1.4)$ \\
\hline Need for blood transfusion & $26(6.7 ; 4.4-9.7)$ & $20(5.9 ; 3.7-9.1)$ & $17(6.7 ; 4-10.5)$ \\
\hline
\end{tabular}

Data are given as absolute numbers with percentages and $95 \%$ confidence intervals (of percentages) in parentheses

Table 4 Primary outcome measure (rate of evidence-based interventions per patient within $6 \mathrm{~h}$ after hospital admission) in the á priori defined sub-groups

\begin{tabular}{llll} 
& Pre-intervention phase & Intervention phase I & $p$ value \\
Children (<15 years) (\%) & $75 \pm 17$ & $79 \pm 15$ & $0.003^{*}$ \\
Adults (\%) & $71 \pm 17$ & $79 \pm 16$ & $<.03^{\#}$ \\
Patients with malaria at hospital admission (\%) & $72 \pm 17$ & $77 \pm 15$ & $<0.001^{*}$ \\
Patients referred from health centers (\%) & $74 \pm 17$ & $79 \pm 15$ & $<0.001^{*}$ \\
\hline
\end{tabular}

Data are given as mean values $\pm S D$

*Significant group difference (unpaired Student's t test)

\# Significant interaction between sub-group and overall treatment effect (regression analysis)

admission (odds ratio per evidence-based intervention implemented, 1.25; 95\% CI 0.96-1.63).

\section{Discussion}

This feasibility trial of 1594 patients found that a focused education program and implementation of an infection treatment bundle increased the rate of early evidencebased interventions in patients with an acute infection treated in a rural, sub-Saharan African hospital. This effect was observed in the entire population as well as in all predefined subgroups. Provision of material resources did not result in a further increase in the rate of early evidence-based interventions provided. Except for an increase in the rate of antimicrobial therapy and fluid resuscitation initiated by health centers, no further secondary end point exhibited statistical significance. We did not observe a difference in the incidence of severe adverse events between the three study phases.

Although the three study phases covered comparable time intervals, the number of patients enrolled in each of the periods differed. Differences in the regional prevalence of acute infectious diseases during the study period can be explained by the two annual rainy seasons in Rwanda, which occur during March and April and during October and November. Given that this trial used specific therapeutic interventions as its primary outcome measure, it is questionable whether these differences have relevantly influenced its results. The education program and acute infection treatment bundle were associated with an increase in the rate of evidence-based interventions performed for patients with acute infections during the first $6 \mathrm{~h}$ after hospital admission. The rate of serious adverse events and the need for blood transfusion remained unchanged during the three study phases.

It should be noted that, before the acute infection education program and bundle implementation, the rate of evidence-based treatment for patients with acute infections was higher than assumed in the power analysis (74 vs. 50\%). Although the investigation does not explain this finding, countrywide implementation of guidelines 
for the management of common infectious diseases may have played a role. In addition, the before-and-after design of our trial may have introduced a Hawthorne effect. The Hawthorne effect has been found to be relatively common in community intervention trials, particularly in resource-limited settings such as sub-Saharan Africa [15].

The high rate of evidence-based treatment in patients with an acute infection before the trial intervention could explain why the difference in the primary outcome measure was small but statistically significant. Nonetheless, the education program and implementation of the infection treatment bundle further increased the rate. This effect was most pronounced in healthcare centers where the incidence of antimicrobial administration and fluid resuscitation rose by 30 and $118 \%$, respectively. Our data highlight the feasibility and value of locally customized education with introduction of a treatment bundle and suggest that such bundles can be effectively introduced even in resource-limited settings.

Surprisingly, consistent provision of material resources to implement the infection treatment bundle in the study hospital and referring health centers (Intervention Phase II) failed to further increase the rate of evidence-based interventions per study patient. This suggests that, even in a resource-limited setting, implementation of an infection treatment bundle is feasible without provision of extra material resources [16-18]. This interpretation is consistent with the results of previous surveys indicating that material resources required to implement the infection treatment bundle (as used in this trial) were widely available even in resource-limited healthcare facilities in middle- and low-income countries [16-18].

The observation that serious adverse events and the need for blood transfusion remained unchanged over the three study phases supports the conclusion that our study interventions were likely safe. Given their low incidence, our trial was underpowered to demonstrate with certainty that implementation of the infection treatment bundle did not result in more adverse events. This conclusion is important because previous sepsis therapies supported by scientific evidence from high-income countries resulted in more complications and/or higher fatality rates when implemented in resource-limited settings [7-9]. For instance, three randomized controlled trials conducted in sub-Saharan Africa reported increased mortality associated with early fluid resuscitation in patients with sepsis and malaria in the absence of mechanical ventilation $[8,9,19]$. The fluid volumes administered in our study were similar to the fluid volumes administered in one of these trials [9] but smaller than the fluid volumes administered in the other two $[8$, 19]. It should also be noted that almost two-thirds of the patients enrolled in our study were children $(<15$ years), and the rate of malaria (co-)infection was high (82.4\%). An important difference between our study and the previous studies was that the patients included in our study demonstrated a lesser severity of illness. In particular, the patients in our study demonstrated less severe anemia. In fact, sepsis criteria were fulfilled in only one in three of our study patients. The fact that fluids were administered at an earlier stage of infection and before cardiovascular compromise may explain why we did not observe a high rate of adverse advents and, in particular, why no pulmonary edema was observed in our study.

The hospital mortality rates observed in our trial were low, which most likely reflects the low overall disease severity of the study population. Both the relatively low rate of HIV infection [20] and the comparatively short duration between symptom onset and presentation to a healthcare facility may have further contributed to the low fatality rate. These observations may reflect the effectiveness of the Rwandese healthcare system and may explain why studies from other sub-Saharan African countries reported higher death rates in patients with acute infection and sepsis [20-22]. Our trial was therefore underpowered to detect a difference in hospital mortality. However, the results of the logistic regression model suggest that the number of evidence-based interventions per patient may be associated with improved hospital survival. Because this association missed the statistical significance level, it should be further evaluated in a larger trial. Similarly, our study was not designed to detect a difference in the incidence of new sepsis occurrence following treatment implementation and hospital admission. We believe, though we cannot provide evidence to confirm, that patient- and disease-related factors, rather than treatment-related factors, determined whether patients fulfilled sepsis criteria only after study inclusion.

Certain limitations must be considered when interpreting the results of this trial. First, antimicrobial resistance is common in sub-Saharan Africa [23] and can crucially reduce the beneficial effects of antimicrobial therapy. Because antimicrobial resistance patterns for the study region were not available, we could not be sure whether antimicrobial therapy as per the study protocol was appropriate and truly effective. Second, our trial was conducted at a single center. This per se increases the risk of inflated estimates as compared to multicenter trials [24]. Furthermore, it is important to notice that site-specific factors (e.g., the absence of an emergency surgery service) and study population-specific factors (e.g., high incidence of malaria) limit the external validity and generalizability of our findings. Third, there is evidence that the effect of specific study interventions (e.g., antibiotic 
therapy, fluid resuscitation) are exquisitely time-sensitive $[25,26]$. Since we only documented whether study interventions were performed during the first $6 \mathrm{~h}$ after hospital admission, we cannot provide data on the exact time points when therapies were initiated. Finally, the fact that only a summary of the study protocol and analysis plan was pre-published (http://www.clinicaltrials.gov), and not a detailed protocol and statistical analysis plan, increased the risk of bias. Notably (and in contrast to the pre-published protocol), we did not measure the exact time point at which study interventions were performed, but we documented whether study interventions were completed during the first $6 \mathrm{~h}$ after hospital admission.

\section{Conclusions}

The results of this feasibility trial indicate that a focused acute infection education program and implementation of an infection treatment bundle increased the rate of early evidence-based interventions in patients with acute infections (mostly malaria) admitted to a rural, sub-Saharan African district hospital. While no safety issues were detected, this could be related to the very low disease severity of the enrolled patient population. The number of early evidence-based interventions might be associated with improved hospital survival.

\section{Electronic supplementary material}

The online version of this article (https://doi.org/10.1007/s00134-018-5266-x) contains supplementary material, which is available to authorized users.

\begin{abstract}
Author details
${ }^{1}$ Gitwe Hospital and Gitwe School of Medicine, Gitwe, Rwanda. ${ }^{2}$ Department of Surgery, California Medical Center, Los Angeles, USA. ${ }^{3}$ Great Lakes Free University, Goma, Democratic Republic of Congo. ${ }^{4}$ Department of Anaesthesia and Critical Care, Makerere University College of Health Sciences, Kampala, Uganda. ${ }^{5}$ Department of Anesthesiology, University of Nebraska Medical Center, Omaha, USA. ${ }^{6}$ Division of Critical Care, Department of Medicine, Charlotte Maxeke Johannesburg Academic Hospital and Faculty of Health Sciences, University of the Witwatersrand, Johannesburg, South Africa. ${ }^{7}$ Division of Pulmonology, Department of Medicine, Charlotte Maxeke Johannesburg Academic Hospital and Faculty of Health Sciences, University of the Witwatersrand, Johannesburg, South Africa. ${ }^{8}$ Seattle Children's Hospital, University of Washington, Seattle, USA. ${ }^{9}$ BC Children's Hospital, University of British Columbia, Vancouver, Canada. ${ }^{10}$ Institute of Medical Statistics, Informatics and Health Economics, Innsbruck Medical University, Innsbruck, Austria. ${ }^{11}$ Society of Critical Care Medicine on behalf of the Surviving Sepsis Campaign, Mount Prospect, IL, USA. ${ }^{12}$ Department of Critical Care Medicine, Mayo Clinic, Phoenix, AZ, USA. ${ }^{13}$ Department of Anesthesiology and Intensive Care Medicine, Kepler University Hospital and Johannes Kepler University Linz, Linz, Austria.
\end{abstract}

\section{Funding}

This study was funded by the Life Priority Fund, the Hellman Foundation, and the King Baudouin Foundation. The research project was supported by the European Society of Intensive Care Medicine and the Society of Critical Care Medicine through the Surviving Sepsis Campaign.

\section{Compliance with ethical standards}

\section{Conflicts of interest}

Dr. Mujyarugamba's institution received funding from Gitwe Hospital. Dr. Nyiringabo received funding from the Society of Critical Care Medicine (SCCM). Dr. Kwizera received travel and accommodation to assist in the training portion of the study. Dr. Bagenda received funding from SCCM and the European Society of Intensive Care Medicine (ESICM); he received support for article research from SCCM and ESICM, and he disclosed having two shares in Mylan NV. Dr. Mer received funding (honoraria for advisory boards and education talks delivered) from Pfizer, AstraZeneca, MSD, Gilead, Aspen, Sun, and 3 M. Ms. Harmon's institution received funding from King Baudouin Foundation $(\$ 25,000)$, Hellman Foundation $(\$ 50,000)$, and BD Corporation (provided supplies of $\$ 5000$ utilized for sepsis carts at Gitwe Hospital in Rwanda and a donation to the project of $\$ 2500$, which was used for the cart purchases and instructional materials). Dr. Farmer received support for article research from the Hellman Foundation. Dr. Patterson received funding from American Board of Anesthesiology, Accreditation Council for Graduate Medical Education, SCCM, Society of Academic Anesthesiology Associations (paid partial expenses after inviting him to give presentations at the Annual Meeting in 2016), and Dickie McCamey LLC (paid expert witness for the defense); he disclosed that his son (Ryan) and daughter (Julia) both received grant funding for projects in Gitwe, Rwanda from the Hellman Foundation (which partially supported this current project via a grant to SCCM); and he received support for article research from the Hellman Foundation. The remaining authors have disclosed that they do not have any potential conflicts of interest.

Received: 1 June 2018 Accepted: 4 June 2018 Published online: 28 June 2018

\section{References}

1. Adhikari NK, Fowler RA, Bhagwanjee S, Rubenfeld GD (2010) Critical care and the global burden of critical illness in adults. Lancet 376:1339-1346

2. Angus DC, van der Poll T (2013) Severe sepsis and septic shock. N Engl J Med 369:840-851

3. Cheng AC, West TE, Limmathurotsakul D, Peacock SJ (2008) Strategies to reduce mortality from bacterial sepsis in adults in developing countries. PLoS Med 5:e175

4. Kissoon N, Uyeki TM (2016) Sepsis and the global burden of disease in children. JAMA Pediatr 170:107-108

5. Dellinger RP, Levy MM, Rhodes A et al (2013) Surviving Sepsis Campaign Guidelines Committee including the Pediatric Subgroup: surviving sepsis campaign: international guidelines for management of severe sepsis and septic shock: 2012. Crit Care Med 41:580-637

6. Schultz MJ, Dünser MW, Dondorp AM et al (2017) Global Intensive Care Working Group of the European Society of Intensive Care Medicine: current challenges in the management of sepsis in ICUs in resource-poor settings and suggestions for the future. Intensive Care Med 43:612-624

7. Maude RJ, Hoque G, Hasan MU et al (2011) Timing of enteral feeding in cerebral malaria in resource-poor settings: a randomized trial. PLoS One 6:e27273

8. Andrews W, Semler MW, Muchemwa L et al (2017) Effect of an early resuscitation protocol on in-hospital mortality among adults with sepsis and hypotension: a randomized clinical trial. JAMA 318:1233-1240

9. Maitland K, Kiguli S, Opoka RO et al (2011) FEAST Trial Group: mortality after fluid bolus in African children with severe infection. N Engl J Med 364:2483-2495

10. Ministry of Health Republic of Rwanda: guidelines. http://www.moh.gov. rw. Accessed 9 Feb 2018

11. Harris PA, Tayler R, Thielke R, Payne J, Gonzalez N, Conde JG (2009) Research electronic data capture (REDCap) — a metadata-driven methodology and workflow process for providing translational research informatics support. J Biomed Inform 42:377-381 
12. Bone RC, Balk RA, Cerra FB et al (1992) Definitions for sepsis and organ failure and guidelines for the use of innovative therapies in sepsis. The ACCP/SCCM Consensus Conference Committee. American College of Chest Physicians/Society of Critical Care Medicine. Chest 101:1644-1655

13. Seymour CW, Liu VX, Iwashyna TJ et al (2016) Assessment of clinical criteria for sepsis: for the Third International Consensus Definitions for Sepsis and Septic Shock (Sepsis-3). JAMA 315:762-774

14. Rahman AE, lqbal A, Hoque DM et al (2017) Managing neonatal and early childhood syndromic sepsis in sub-district hospitals in resource-poor settings: improvement in quality of care through introduction of a package of interventions in rural Bangladesh. PLoS One 12:e0170267

15. Barnes BR (2010) The Hawthorne effect in community trials in developing countries. Int J Soc Res Method 13:357-370

16. Baelani I, Jochberger S, Laimer T et al (2012) Identifying resource needs for sepsis care and guideline implementation in the Democratic Republic of the Congo: a cluster survey of 66 hospitals in four eastern provinces. Middle East J Anaesthesiol 21:559-575

17. Bataar O, Lundeg G, Tsenddorj G et al (2010) Helfen Berührt Study Team: nationwide survey on resource availability for implementing current sepsis guidelines in Mongolia. Bull World Health Organ 88:839-846

18. Baker T, Lugazia E, Eriksen J, Mwafongo V, Irestedt L, Konrad D (2013) Emergency and critical care services in Tanzania: a survey of ten hospitals. BMC Health Serv Res 13:140

19. Andrews B, Muchemwa L, Kelly P, Lakhi S, Heimburger DC, Bernard GR (2014) Simplified severe sepsis protocol: a randomized controlled trial of modified early goal-directed therapy in Zambia. Crit Care Med 42:2315-2324
20. Kwizera A, Nabukenya M, Peter A et al (2016) Clinical characteristics and short-term outcomes of HIV patients admitted to an African intensive care unit. Crit Care Res Pract 2016:2610873

21. Jacob ST, Moore CC, Banura P et al (2009) Promoting Resource-Limited Interventions for Sepsis Management in Uganda (PRISM-U) Study Group: severe sepsis in two Ugandan hospitals: a prospective observational study of management and outcomes in a predominantly HIV-1 infected population. PLoS One 4:e7782

22. Huerga $H$, Vasset B, Prados E (2009) Adult and paediatric mortality patterns in a referral hospital in Liberia 1 year after the end of the war. Trans $R$ Soc Trop Med Hyg 103:476-484

23. Agaba P, Tumukunde J, Tindimwebwa JVB, Kwizera A (2017) Nosocomial bacterial infections and their antimicrobial susceptibility patterns among patients in Ugandan intensive care units: a cross sectional study. BMC Res Notes 10:349

24. Bafeta A, Dechartres A, Trinquart L, Yavchitz A, Boutron I, Ravaud P (2012) Impact of single centre status on estimates of intervention effects in trials with continuous outcomes: meta-epidemiological study. BMJ 344:e813

25. Seymour CW, Gesten F, Prescott HC et al (2017) Time to treatment and mortality during mandated emergency care for sepsis. N Engl J Med $376: 2235-2244$

26. Leishman D, Wie B, Doerfler M et al (2016) Association of fluid resuscitation initiation within 30 minutes of severe sepsis and septic shock recognition with reduced mortality and length of stay. Ann Emerg Med 68:298-311 